\title{
An approach to investigating the capability of manufacturing equipment for handling food product variation
}

\author{
Jason Matthews, Baljinder Singh, Glen Mullineux, Lian Ding and Tony Medland
}

Department of Mechanical Engineering, University of Bath, Bath, BA2 7AY, UK

\begin{abstract}
Commercial pressures in the UK mean food companies must continually reinvent and evolve their products, creating large product families. The ability to handle both the complexity of processes and large variations in product format, leads to considerable difficulties in ensuring that manufacturing and packaging equipment can firstly handle the product, and secondly manufacture it efficiently. This paper presents an approach built on the understanding of the relationships between food product properties and processing parameters. The approach can assist the engineer to redesign the processing equipment from knowing the properties of the product characteristics, and also to reverse engineer the product from the bounds of the process, with the goal of producing a capable and efficient process. The potential for the methodology and its application are summarised for industrial case studies on confectionery carton erection, and the 'late customisation' of yoghurt with different fruits.
\end{abstract}

Keywords: food product variation, packaging equipment design, food processing equipment design, manufacturing efficiency, late customisation, carton erection machines

\section{Background}

\subsection{Process and product overview}

Previous research has identified that the food processing industry maintains the highest number of product variations and makes more product changes than any other mass-producing industry (Fischer et al. 2005). Many of these arise over short periods due to marketing and customer demands; some products are stable over long periods, whilst others are short lived or seasonal. The complexity of food production is further increased by the diverse nature of such products which range from large solids to liquids and pastes. Their processing is also diverse, from simple assembly processes of liquids and solids through to the control of complex chemical and cooking processes. It is common within the industry for manufacturers to run their equipment at decreased efficiencies in order to meet customer demands for a new or variable product. The ability to handle both the complexity of the process and large variations in product format creates extreme difficulties in ensuring that the manufacturing, handling and packaging equipment can cope with such factors.

\subsection{Aims and approach}

The work presented in this paper was the result of a three year collaborative industrial research project based at the

Food Manufacturing Efficiency 1 (3) 1-7. DOI: 10.1616/1750-2683.00xx ISSN 1750-2683 C IFIS Publishing 2007. All Rights Reserved
University of Bath to investigate the capabilities of food processing and packaging equipment in handling product variation. The goal was to create a methodology and supporting tools to determine the ability of existing plants to handle new variations of the product at an early development stage. Such a methodology allows the development team to establish whether the existing plant is adequate, whether a new plant needs to be created or whether slight changes in product specification or form will allow the existing plant to be utilised. The approach is based upon an understanding of the food product feature characteristics, together with an understanding of the equipment capabilities. The paper also presents research findings and includes a taxonomy of food product-process relationships, which can be used to model the product. In addition to this, the limiting factors of food processing equipment are identified; these factors must be implemented in the modelling and simulation of the equipment.

The equipment investigated is limited to the actual processing and packaging equipment employed in the food industry.

- For processing operations, this includes: mechanical mixing, agglomerations, mincing/slicing, transferring, weighing and counting, cooking, and freezing

- For packaging operations this includes: cartoning, sealing and tucking, over-wrapping and bagging.

Pre-processing equipment has not been considered at this stage. 
As with any design activity, a range of questions arises about the parameters involved. For the specific problems examined in this research, the following factors were identified:

- Food product properties

Which are important?

- How fixed are they?

- Do they change with season?

- Do they change with ingredient variety?

$\circ$ Do they change with processing? If so, do they recover?

- Consumer requirements

- Sensory properties such as taste, texture

- Are these sensory properties constant?

- How do the sensory properties map onto physical properties?

- Availability of appropriate experimental models

- Many models proposed

$\circ$ How good are these models?

$\circ$ How relevant are the models?

This paper presents how these parameters can be fitted into a methodology, for both the evaluation and redesigning of food processing equipment to handle a variable product. Section 2 identifies the factors that relate to the food products processed. Section 3 discusses how the equipment is dealt with. Section 4 presents the methodology, and Section 5 summarises the implementation of the methodology in two case studies.

\section{Food product characteristics}

Due to the handling and transfer processes involved, key food characteristics, such as strength and resistance to damage, or movement upon a conveyor belt, may need to be assessed. Many of these characteristics need to be determined and studied if the capability of the plant to handle such products is to be understood. Foodstuffs differ from most commercial manufactured products in the fact that customer perception of the product is a very impor- tant factor. Since customers' views of quality comes from their senses, manufacturers use sensory panels to assess product quality. The information obtained from equipment and product data is then used to model effects of product variation on processing equipment (Huda and Chung 2002).

\subsection{Product variation findings}

Investigation into the raw materials that the food industry processes shows they can be categorised into five types: liquids, pastes/slurries, particulates, and solids (which may be either rigid or soft). Examples of products that fit into these categories can be seen in Table 1 .

Table 2 shows a sample of variable product changes which the food industry has to cope with. These and variational changes can be divided into nine distinct categories:

- Increase in product size

- Change in packaging density

- Constituent change

- Raw material size variation

- Physical properties of product change

- Change in packaging materials

- Percentage increase in product per container

- Environmental factors

- Organic product change.

\subsection{Limiting factors}

The results shown in Table 2 are just a few examples which the food processing industry has to handle throughout the life of a product. When looking at Table 2, it can be seen that several generic product limiting factors arise. These factors include: density, weight, geometric size, tolerance, shape and mechanical properties. Within the product processing context there is a direct and logical link between geometric size, tolerance and shape. The boundary values for such factors have normally been acquired through manufacturers' experiences, but in some cases component specific testing is required (e.g. shear strain testing of stirred yoghurt products).

Table 1. Types of raw materials used in food processing

\begin{tabular}{|c|c|c|c|c|}
\hline \multirow[b]{2}{*}{ Liquids } & \multirow[b]{2}{*}{ Paste/Slurry } & \multirow[b]{2}{*}{ Particulates } & \multicolumn{2}{|c|}{ Solids } \\
\hline & & & Rigid & Soft \\
\hline Milk & Yoghurts & Coffee & Chocolate & Bread \\
\hline Soft drinks & Fish pastes & Sauce granules & Cookies & \\
\hline Beverages & Yellow spreads & Tea & Frozen vegetables & Cakes \\
\hline \multirow[t]{2}{*}{ Soups } & Jams & Cake mixes & & Meats \\
\hline & & Pasta & & Jellies \\
\hline
\end{tabular}


Table 2. Effects of product variation on food processing equipment

\begin{tabular}{|c|c|c|}
\hline Type of variation & Industrial Examples of Problem & System effects \\
\hline Increase in product size & $\begin{array}{l}\text { Change in product dimensions for } \\
\text { over-wrapping. }\end{array}$ & Geometric, kinematic, dynamic, tolerance \\
\hline Change in packaging density & Two extra frozen puddings per pack. & Geometric, kinematic, volumetric \\
\hline Constituent change & $\begin{array}{l}\text { Customer product variation may force the } \\
\text { manufacturer to expand range. The addition of } \\
\text { noodles and croutons to dried soup range. }\end{array}$ & Dynamic, geometric, weight, density, tolerance \\
\hline Raw material size variation & $\begin{array}{l}\text { Potatoes sliced for crisps etc. Factors governing } \\
\text { raw materials, such as potato shape, cannot be } \\
\text { guaranteed, and can only be graded to a general } \\
\text { standard. }\end{array}$ & Kinematic, geometric, dynamic, shape, tolerance \\
\hline $\begin{array}{l}\text { Physical properties of } \\
\text { product change }\end{array}$ & $\begin{array}{l}\text { Shifting from transferring fruit cake to a soft } \\
\text { cream cake or pie. Softer product less resistant } \\
\text { to higher kinematics and dynamics. }\end{array}$ & Kinematic, dynamic \\
\hline Change in packaging materials & $\begin{array}{l}\text { Environmental regulations are forcing } \\
\text { manufacturers to move towards thinner and } \\
\text { biodegradable packaging materials. }\end{array}$ & Kinematic, mechanical properties \\
\hline $\begin{array}{l}\text { Percentage increase in product } \\
\text { per container }\end{array}$ & $30 \%$ extra cereal in a carton. & Geometric, density, weight \\
\hline Environmental factors & $\begin{array}{l}\text { Humidity can change the folding properties of } \\
\text { carton skillets. Carton often stored away from } \\
\text { product area, this can affect setting of machine. }\end{array}$ & Kinematic, mechanical properties \\
\hline Organic product change & $\begin{array}{l}\text { The physical properties of potatoes change over } \\
\text { the picking season; this has an effect on } \\
\text { processing equipment. }\end{array}$ & Kinematic, shape \\
\hline
\end{tabular}

\subsection{Food product-process relations}

Figure 1 shows relationships between the properties of foodstuffs, process effects and the limiting factors identified in section 2.2.

Column three of Figure 1 outlines the relationship constraints of the product. These are the key factors that affect the ability of any system to process variable products:

- Geometric relationships are indispensable for each feature, which have a standard range for specifying the value of each parameter for the shape and geometric size. Shape and geometric structure of the product is important when considering retentions for grippers and transfer guides.

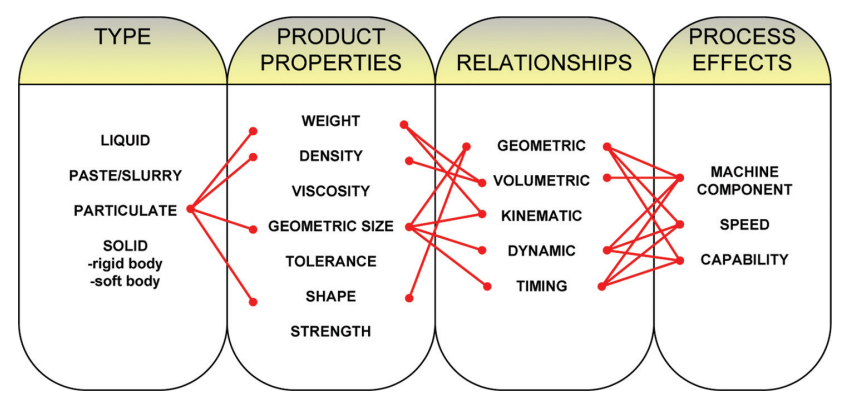

Figure 1. Food product-processing relationships.
- Kinematic relationships are especially important when considering the transfer of product.

- Dynamic relationships are important considerations as the product mass increases, the forces applied will also increase.

- Volumetric relationships are very similar to geometric constraints, except that the area/volume of the product is considered important when product is retained by the manufacturing system and when product has to be put into containers and packaging.

- Timing relationships represent the fact that the ability to move product changes as factors such as weight and size change with the variable product.

For effective analysis of the potential processing of a variable product, any model used must have the ability to handle/process these relationships. Column four of Figure 1 shows the factors of the processing ability that are influenced. The research shows a distinct relationship between the food product features identified earlier, and their effects on the system. Figure 1 shows these relationships when considering processing for production of gravy granules, a particulate product.

\section{Processing equipment capability and efficiency}

For the assessment of product variables on equipment, the critical factor is the identification and formalisation of the 
functional requirements for the design, with respect to the inherent capabilities of the existing design. This inherent capability affects manufacturing efficiency, which, in turn, influences time, flexibility and quality dimensions - such that rapid product throughput, high adaptability and low product defect counts are routinely measured. However, for the majority of manufacturers, the most important factor associated with manufacturing efficiency is the level of total cost incurred. If the costs to produce a similar, but variable product increases, then from the manufacturer's viewpoint, the efficiency has decreased. With the functional requirements for the processing equipment specified, the constraints imposed by the existing equipment and that of the variable product can be formalised for the design problem. There are two types of models that have been extensively used in the modelling of manufacturing systems: prescriptive and descriptive. Prescriptive models are generally employed to construct decisions on that system, while descriptive models are mostly used for performance evaluation of the manufacturing system. These models can be sub-categorised into analytical and simulation models. The following section highlights techniques for physical form modelling for food equipment.

\subsection{Form modelling and simulation of the physical system}

Modelling and simulation analyses are well established techniques for analysing the potential effects of complex manufacturing changes. The benefits of modelling the processing of a product for the food industry has been demonstrated by Wedzicha and Roberts (2007). There are also several reasons why such approaches should be employed to investigate the capabilities of industrial machinery to process variable products:

- It gives the manufacturer the opportunity to experiment and analyse in a relatively low cost and low risk environment.

- In an industrial environment, the cost of getting it wrong is often very high and carries high risk. For example, equipment damage has the dual expense of replacement parts and lost production capability.

- Changes to the real system are sometimes expensive, difficult or even impossible to achieve in today's manufacturing environments.

As noted by Huda and Chung (2002), the specific manufacturing process employed in the food industry initially requires a continuous event modelling approach, and then later a discrete event approach. One approach that has been employed to access the design capability of food processing equipment using the identified bounds of the manufacturing system is limits modelling (Matthews et al.
2006). The developed approach employs a parametric model of the system defined within a constraint-modelling environment. The information used to produce the model is generated from machine drawings (if available), manual measurements and high speed video. The high-speed video is also used to validate that the model represents reality. Failure modes for the model are derived from testing of the product to be manufactured and by a consensus of the designer and manufacturer. Parametric variation is employed to 'disturb' the geometry of the mechanism, and the model is then actuated. Constraint monitoring is employed to check if the model violates any of the applied constraints (failure modes).

The successful configuration returned from functioning instances are used to produce the functional matrix. The values from this matrix can then be visually represented to produce the performance envelope for the equipment. Interrogation of these representations allows the engineer to see if a variable product can be produced using the modelled equipment. When simulating and modelling the processing equipment with methods such as that presented in Matthews et al. (2006), what has become evident is that there are six generic limiting factors that have to be handled with any modelling approach: element collision, incorrect construction, mechanism deconstruction, displacement, dynamics and kinematics (velocity, acceleration and jerk). An explanation of these limiting factors is given in Table 3. Missing from the table is the incorrect construction' factor. This is specific to modelling approaches that use rule-based strategies for their modelling and simulation. Since models are constrained to assembly, and satisfy the given rules, the outputted assembly may not be the same as the object being modelled. An example of this is commonly seen with the four bar mechanism, which can be assembled in an inverse manner, even though, as far as the modeller is concerned, the constraints are met.

\section{Proposed methodology}

The major factor that has been identified in this research is that, although there is a vast amount of research on food product properties, and some published research on food processing equipment, there is a lack of research that combines the use of both for the handling of variable products. The following is a proposed approach to handle such a problem. The flowchart in Figure 2 shows the proposed methodology presented in this paper. What is immediately identifiable is that it is a concurrent procedural process that identifies the key characteristics of both product and process. Once established, their relative boundaries and limits are identified; these are then used to produce specified models of product and process. With these models established, the effects of product variation can be opti- 
Table 3. Limiting factors involved in simulating/ modelling processing equipment

\begin{tabular}{ll}
\hline Limiting factor & Description \\
\hline Element collision & $\begin{array}{l}\text { Clash interaction between elements } \\
\text { of equipment } \\
\text { Mechanism } \\
\text { deconstruction } \\
\text { Displacement }\end{array}$ \\
$\begin{array}{l}\text { pull apart } \\
\text { Too much or insufficient movement of } \\
\text { element to translate required motion }\end{array}$ \\
Velocity & $\begin{array}{l}\text { Low or high velocities can cause } \\
\text { timing problems }\end{array}$ \\
Acceleration & $\begin{array}{l}\text { Excessive acceleration and jerk } \\
\text { cause vibration }\end{array}$ \\
Lerk & $\begin{array}{l}\text { Lack of accuracy and advanced wear } \\
\text { Effects of forces on the motion, increase } \\
\text { in speed and product load can cause } \\
\text { vibrations, increased wear and lack of } \\
\text { accuracy }\end{array}$ \\
\hline
\end{tabular}

mised to find either the best product or process solution. It is then used to answer the questions:

- Can a given process deal efficiently with the food variation?

- Can a given process variant deal with existing food?

- Can an optimal arrangement be found?

\section{Industrial case studies}

The methodology has been implemented using two different case studies, a food packaging set-up and a food processing set-up. The case studies show how the identification of a system's bounds can be employed to model the effects of product variation, and the finding of a solution which will accept this variation.

\subsection{Carton erection}

Carton erection machines are used in the food industry to produce carton boxes. Examples of such products can be seen in the secondary packaging of confectionery, such as boxes of sweets. In this case study, the requirement was to increase production throughput, which meant increasing the speed of the carton erection machine. However, it was found that the peak velocity achieved caused many of the cartons to be torn.

In operation, the machine was loaded with a stack of pre-cut and pre-creased board nets. In each cycle, one board net is transferred from the stack and placed over the opening of a die. A plunger then carries the carton vertically downwards through a die section. This has the effect of folding up the walls of the box, thus erecting the

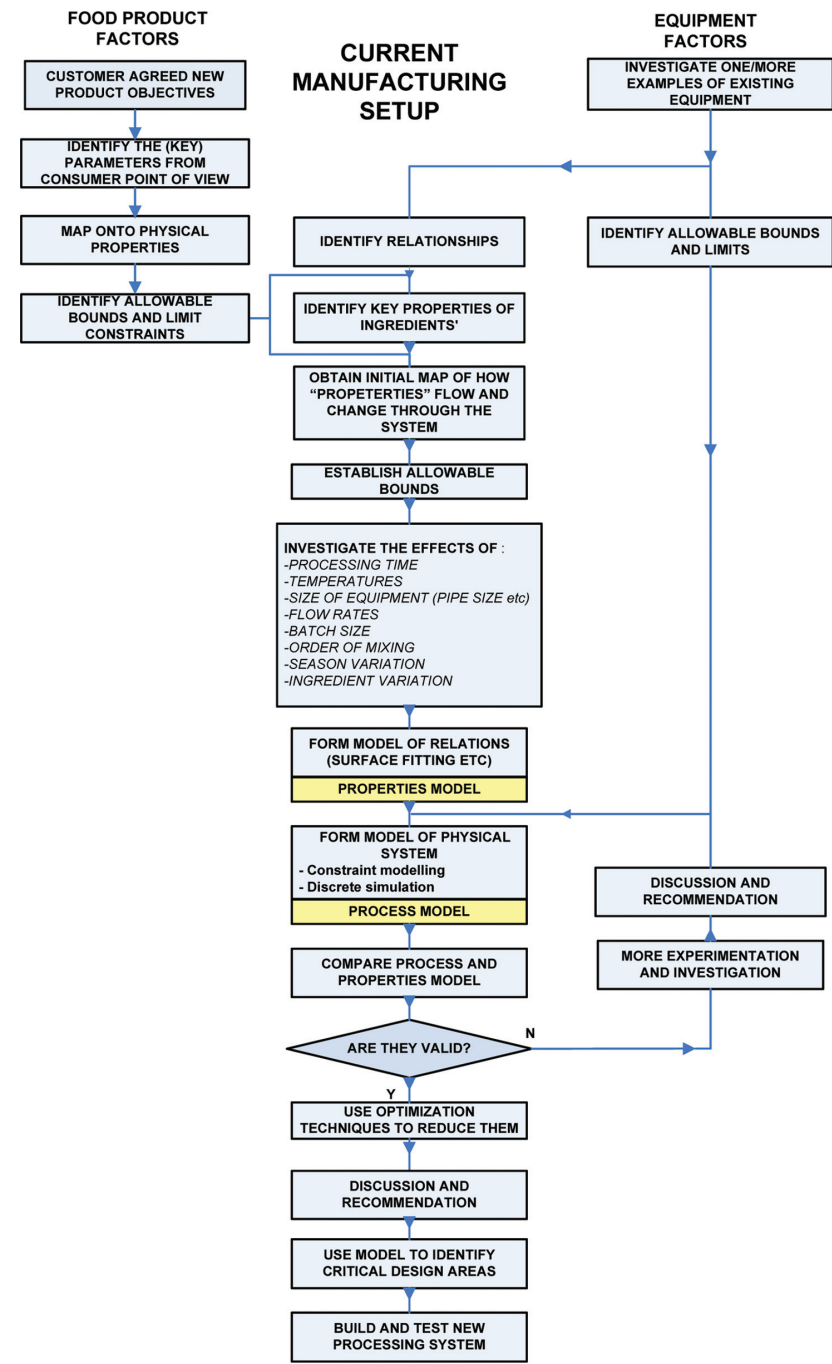

Figure 2. Proposed methodology involved in modelling the effects of product variation on food processing equipment.

carton. Figure 3a shows a two-dimensional representation of the side view for the carton erecting machine. Figure $3 b$ shows a three-dimensional model of the machine which is driven by a single motor.

At increased speed, the output of the machine was limited by the mechanical features of the carton, in particular the ability of the carton to withstand the impact without sustaining damage, such as tearing or delamination. Under test at higher speeds, it was found that the reciprocating action of the plunger impacted the board at its maximum speed (peak velocity).

A constraint modelling environment (Mullineux 2001) was employed to produce a form model of the physical system (see Figure 3c) and an additional link was added to the design with one end constrained to move along a linear track. To achieve this, the lengths of the links were allowed to vary, along with the offset position of the joint 
(a)

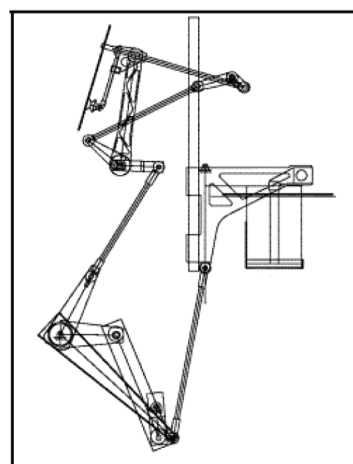

(b)

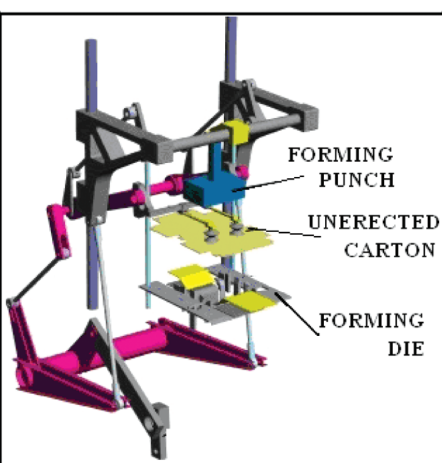

(c)

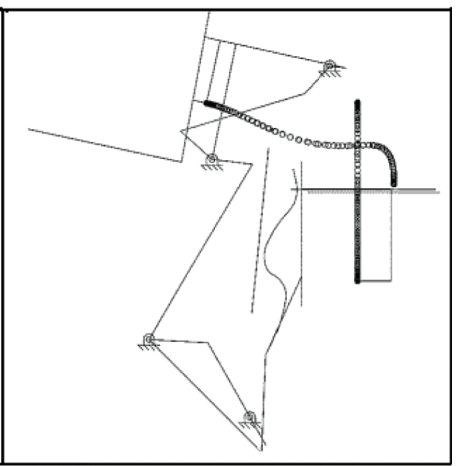

Figure 3. Carton erector system. (a) A two-dimensional representation of the side view of the carton erecting machine. (b) A three-dimensional model of the machine. (c) A form model of the physical system, using a constraint modelling environment.

(with respect to the original link) with a view to reducing the peak velocity. Thus, the model analysis showed at what position the peak velocity was being achieved, and this enabled a re-design of the equipment from a single crank mechanism to a split crank mechanism. With models established, it was then possible to evaluate other variable cartons.

In theory, the models showed that a $23 \%$ increase in throughput could be achieved when the system was redesigned. However, it was found that when the speed was reduced substantially, the shape of the cam track was unacceptable because of considerations of pressure angle. The inclusion of constraints relating to the cam laws meant that in practice only a $10 \%$ reduction in impact velocity could be achieved. Figure 4 therefore shows that the relationships identified earlier remain true.

\subsection{Stirred yoghurt processing}

In the second case study, there were two requirements. First, to optimise the configuration of process pipes in terms of pumping pressures, pipe diameters and lengths, in order to optimise temperature control and reduce pro-

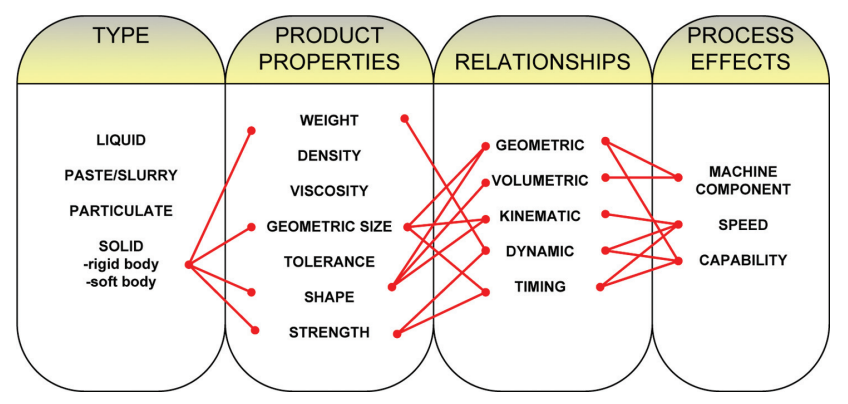

Figure 4. Food product-processing relationships as applied to the carton erector case study. duct damage; second, to enable the 'late customisation' of the base yoghurt with different types of fruits and flavours (see Figure 5).

The variation in yoghurt stems from its consistency and the addition of flavourings and fruits pulps. Yoghurt is a non-Newtonian material and is thixotropic so that work performed on it results in shear thinning. Whilst there is some recovery (over a period of time), the aim is often to try to minimise the amount of processing that is carried out upon the product. The amount of work required to pump and mix depends upon the temperature.

There is a trade-off between the ease of processing (and reduction in damage) and the need to keep the temperature low in the interests of maintaining product quality during storage, until a decision has been made on which fruit type is to be added. One option was to undertake the processing at room temperature and only cool the product in the pots after filling. An alternative was to cool in the pipe as the product was being moved into the fruit/flavour filling station.

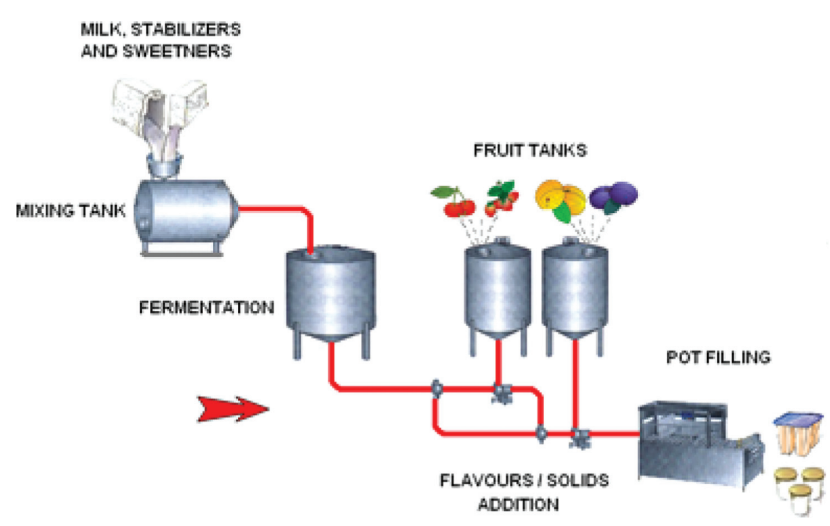

Figure 5. Schematic representation of a yoghurt processing plant. 


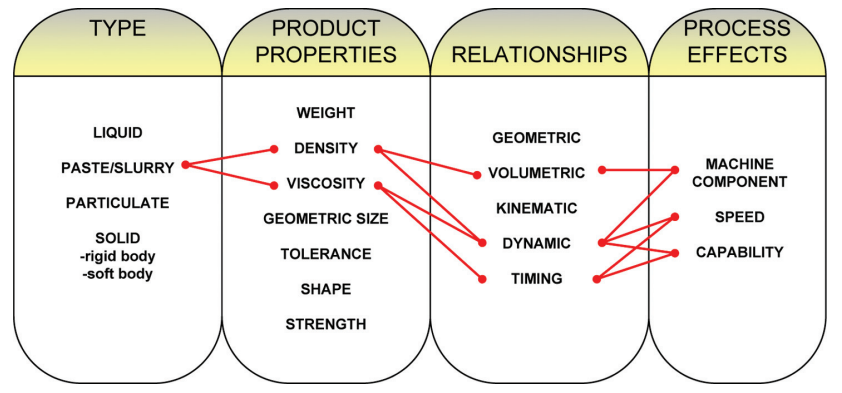

Figure 6. Food product-processing relationships as applied to the yoghurt processing case study.

Given the conflicting requirements, at the form modelling stage, a constraint-based modelling approach was well-suited to looking for an optimal design of the production system. The main difficulty is that the properties of yoghurt do not seem to be well understood. A number of rheological models have been proposed (Holdsworth 1993) for various foodstuffs. These include the HerschelBulkley model (Herschel and Bulkley 1926), the Power law (Da Waele 1923), and Cross's model (Cross 1965). While these have all been used to model yoghurt, they do not account for the parameters of time and temperature, which are essential given the nature of the product.

To cope with this, a model was proposed by Mullineux and Simmons (2006). This model was employed to investigate both product and process. Although a yoghurt producer would prefer to reduce costs of the pumping rigs by reducing pipe size, the optimisation process showed detrimental effects to the product. It was also shown in this process that the pressures required to pump the yoghurt could not be achieved until a relatively large pipe radius was used.

Figure 6 shows that the relationships identified earlier remain true. They identify to the designer which relationships must be dealt with and the effects on the processing system

\section{Conclusion}

This paper describes an approach which offers the design engineer of food processing equipment the ability to:

- Redesign the processing equipment from knowing the properties of the product features

- Reverse engineer the product from the bounds of the process

- Optimise the two options above for efficient manufacture.
The paper also presents a taxonomy of foodstuffs, and a taxonomy of food product-process relationships, which can be used to model the product. In addition to this, the limiting factors of food processing equipment are identified which must be considered in the modelling and simulation of the equipment. The successful application of the approach has been summarised for two industrial case studies.

\section{Acknowledgments}

The work reported was supported by the Department of Trade and Industry and the Department for Environment Food and Rural Affairs (DEFRA), Food Processing Faraday Knowledge Transfer Network, and as part of the Engineering and Physical Sciences Research Council (EPSRC) and Innovative Manufacturing Research Centre at the University of Bath. The research involved a number of industrial collaborators. The authors gratefully express their thanks for the advice and support of all concerned.

\section{References}

Barton, P.I. and Lee, C.K. 2002. Modelling, simulation, sensitivity analysis and optimization of hybrid systems. ACM Transactions on Modelling and Computer Simulation 12(4): 256-289.

De Waele, A. 1923. Viscometry and plastometry. Journal of the Oil Colour Chemists Association 6: 33-31.

Cross, M.M. 1965. Rheology of non-Newtonian fluids: a new flow equation for pseudo-plastic fluids. Journal of Colloid Science 20: 417-427.

Fisher, C., Medland, A.J. and Mullineux, G. 2005. Design for food quality. Proceedings of the 15th International Conference on Engineering Design (ICED05). Melbourne, Australia.

Herschel, W.H and Bulkley, R. 1926. Konsistenzmessungen von Gumni-Benzollosungen. Kolloid Zeitschrift 39: 291-300.

Holdsworth, S.D. 1993. Rheological models used for the prediction of flow properties of food stuffs. Trans IChemE part $C$ 71: 139-179.

Huda, A.M. and Chung, C.A. 2002. Simulation modelling analysis issues for high-speed combined continuous and discrete food industry manufacturing processes. Computers and Industrial Engineering 43: 473-483.

Matthews, J., Singh, B., Mullineux, G. and Medland, A.J. 2006. A constraint-based approach to investigate the "process flexibility' of food processing equipment. Journal of Computers and Industrial Engineering 51(4): 809-820.

Mullineux, G. 2001. Constraint resolution using optimization techniques. Computers and Graphics 25(3): 483-492.

Mullineux, G. and Simmons, M.J.H. 2006. Effects of processing on shear rate of yoghurt. Journal of Food Engineering 79: 850-857.

Wedzicha, B. and Roberts, C. 2007. Modelling: a new solution to old problems in the food industry. Food Manufacturing Efficiency 1(1): 1-8. 